\title{
Estimating the Variance of an Exponential Distribution in the Presence of Large True Observations
}

\author{
Housila P. Singh and Vankim Chander \\ School of Studies in Statistics, Vikram University, Ujjain, India
}

\begin{abstract}
The present paper discusses some classes of shrinkage estimators for the variance of the exponential distribution in the presence of large true observations when some a priori or guessed interval containing the variance parameter is available from some past experiences. Empirical study shows the high efficiency of the developed classes of shrinkage estimators when compared with Pandey and Singh's estimator, minimum MSE estimator and special classes of shrinkage estimators.
\end{abstract}

Zusammenfassung: Dieser Aufsatz diskutiert einige Klassen von shrinkage Schätzern für die Varianz der Exponentialverteilung falls große wahre Beobachtungen vorhanden sind und falls ein priori oder mutmaßliches Intervall aus vergangener Erfahrung verfügbar ist, das diesen Varianzparameter enthält. Eine empirische Studie zeigt die Effizienz dieser Klasse von Schätzern verglichen mit dem Schätzer von Pandey und Singh, dem Minimum MSE Schätzer und speziellen Klassen von shrinkage Schätzern.

Keywords: Bias, Guessed Interval, Mean Squared Error, Percent Relative Efficiency.

\section{Introduction}

The exponential distribution has its significance due to its variety of applications in reliability engineering and life testing problems. The exponential distribution would be an adequate choice for a situation where failure rate appears to be more or less constant. The problem considered in this paper can be illustrated by the question that a sampler frequently asks himself, particularly if he is working with relatively small samples. The question is, "what do I do with large or extreme observations in the sample?" The sampler first attempt to answer this question by a careful review of the data to see if an outlier has somehow appeared or if in fact the offending observation or observations are actually true observations. It is also noted that in practice the experimenter often possesses some knowledge of the experimental conditions, based on awareness with the performance of the system under investigation or from the past experience or from some extraneous source and thus in opinion to give an adequate guessed interval of the value of the variance. In this paper we suggest some classes of shrinkage estimators for the variance of exponential distribution in the presence of large true observations when some a priori or guessed interval $\left(\theta_{1}^{2}, \theta_{2}^{2}\right), \theta_{1}^{2}<\theta_{2}^{2}$, containing the parameter $\theta^{2}$, say, is available from some past experiences.

Let $x_{1}, \ldots, x_{n}$ be a random sample of size $n$, drawn from the exponential distribution. The probability density function of which is given by

$$
f(x ; \theta)= \begin{cases}\frac{1}{\theta} \exp (-x / \theta) & x \geq \theta, \theta>0 \\ 0 & \text { otherwise }\end{cases}
$$


where $\theta$ is the mean and acts as a scale parameter and $\theta^{2}$ is the variance.

Pandey and Singh (1977) suggested the minimum mean squared error (MMSE) estimator

$$
\hat{\theta}_{\text {MMSE }}=\frac{n^{2}}{(n+2)(n+3)} \bar{x}^{2}
$$

for $\theta^{2}$ in the class of estimators of the form $M \bar{x}^{2}$, where $M$ is a suitably chosen constant and $\bar{x}=\sum_{i=1}^{n} x_{i} / n$. The bias and mean squared error (MSE) of $\hat{\theta}_{\text {MMSE }}$ are

$$
\operatorname{bias}\left(\hat{\theta}_{\text {MмSE }}\right)=-\frac{2(2 n+3) \theta^{2}}{(n+2)(n+3)}
$$

and

$$
\operatorname{MSE}\left(\hat{\theta}_{\text {MMSE }}\right)=\frac{2(2 n+3) \theta^{4}}{(n+2)(n+3)} .
$$

Tracy et al. (1996) envisaged a class of shrinkage estimators for $\theta^{2}$ when some point prior information $\theta_{0}^{2}$ of $\theta^{2}$ is available, and is given as

$$
\xi_{(h)}=\theta_{0}^{2}+\alpha_{(h)}\left(\bar{x}^{2}-\theta_{0}^{2}\right),
$$

where

$$
\alpha_{(h)}=n^{2 h} \frac{\Gamma(n+2 h)}{\Gamma(n+4 h)}
$$

and $h$ is a non-zero real number. In particular, if $h=1$ then $\xi_{(h)}$ reduces to the point estimator for the variance, which is given as

$$
\xi_{(1)}=\theta_{0}^{2}+\frac{n^{2}\left(\bar{x}^{2}-\theta_{0}^{2}\right)}{(n+2)(n+3)},
$$

and is due to Tracy et al. (1996).

The distribution (1) is positive valued and positive skewed. It has positive probability that the sample may contain one or more observations from right tail of the distribution leading to a larger estimate of the parameter using unbiased estimator. In such a situation, where some "extremely large" values $x_{i}>t$ are present in the sample, Searls (1966) suggested an estimation procedure suitable to estimate the population mean $\theta$, which reduces the effect of such large true observations for the distribution which is unimodal, positive valued, and positively skewed. Searls (1966) defined the estimator for $\theta$ as

$$
\bar{x}_{t}=\frac{1}{n}\left(\sum_{j=1}^{m} x_{j}+(n-m) t\right), \quad m=0,1, \ldots, n, x_{j} \leq t,
$$

which is formulated by replacing all the observations greater than a predetermined cutoff point $t$ by the value of $t$ itself. Searls (1966) has shown that there exists a wide range of $t$ values for which the MSE of $\bar{x}_{t}$ is less than the variance of the usual unbiased estimator $\bar{x}$. We also refer to Bartholomew (1957), Ojha and Srivastava (1979), Ojha (1982), Srivastava et al. (1985), Srivastava (1986), Singh (1987), Srivastava and Kumar (1990), Upadhyaya et al. (1997), and Singh and Shukla (2002) in this context. 
The estimation problem using some a priori information regarding some population parameters has been investigated by various authors, for example see Mehta and Srinivasan (1971), Jani (1991), Singh and Saxena (2003), Saxena and Singh (2004), Singh et al. (2004), Singh and Saxena (2005), Saxena (2006), Saxena and Singh (2006), and also Singh and Chander (2007).

Thompson (1968) considered the problem of shrinking an unbiased estimator $\hat{\psi}$ of $\psi$ towards an interval $\left(\psi_{1}, \psi_{2}\right)$ and suggested a shrinkage estimator $\hat{\psi}+(1-p)\left(\psi_{1}+\psi_{2}\right) / 2$, where $0 \leq p \leq 1$ is constant. The objective is to propose a class of shrinkage estimators of $\theta^{2}$, when a prior or guessed interval of $\theta^{2}$ is available in the form of $\left(\theta_{1}^{2}, \theta_{2}^{2}\right), \theta_{1}^{2}<\theta_{2}^{2}$.

\section{The Suggested Classes of Shrinkage Estimators}

Let the prior information on $\theta^{2}$ be available in form of an interval with end points $\theta_{1}^{2}$ and $\theta_{2}^{2}$, where $\theta_{1}^{2}<\theta_{2}^{2}$. The arithmetic mean (AM), the geometric mean (GM), and the harmonic mean (HM) are measures of location, which are used for suggesting different classes of shrinkage estimators for $\theta^{2}$ in model (1). We define the family

$$
\tilde{\xi}_{(h)}^{(i)}=A G H(a, b)+\alpha_{(h)}\left(\bar{x}^{2}-A G H(a, b)\right),
$$

where $\alpha_{(h)}$ is given by (4) and $h$ is a non-zero real number. Moreover,

$$
A G H(a, b)=\left(\theta_{1}^{2} \theta_{2}^{2}\right)^{a}\left(\frac{1}{2}\left(\theta_{1}^{2}+\theta_{2}^{2}\right)\right)^{b},
$$

and $i=1,2,3$ corresponds to pairs $(a, b)$ respectively taken as $(0,1),(1 / 2,0)$, or $(1,-1)$ in $A G H(a, b)$.

It is interesting to note that

- for $(a, b)=(0,1)$ in $(6)$ we get a class of shrinkage estimators based on the arithmetic mean $\left(\theta_{1}^{2}+\theta_{2}^{2}\right) / 2$, defined as

$$
\tilde{\xi}_{(h)}^{(1)}=\frac{1}{2}\left(\theta_{1}^{2}+\theta_{2}^{2}\right)+\alpha_{(h)}\left(\bar{x}^{2}-\frac{1}{2}\left(\theta_{1}^{2}+\theta_{2}^{2}\right)\right),
$$

- for $(a, b)=(1 / 2,0)$ we get a class of shrinkage estimators based on the geometric mean $\sqrt{\theta_{1}^{2} \theta_{2}^{2}}$, given as

$$
\tilde{\xi}_{(h)}^{(2)}=\sqrt{\theta_{1}^{2} \theta_{2}^{2}}+\alpha_{(h)}\left(\bar{x}^{2}-\sqrt{\theta_{1}^{2} \theta_{2}^{2}}\right),
$$

- for $(a, b)=(1,-1)$ we get a class of shrinkage estimators based on the harmonic mean $2 \theta_{1}^{2} \theta_{2}^{2} /\left(\theta_{1}^{2}+\theta_{2}^{2}\right)$, defined as

$$
\tilde{\xi}_{(h)}^{(3)}=2 \frac{\theta_{1}^{2} \theta_{2}^{2}}{\theta_{1}^{2}+\theta_{2}^{2}}+\alpha_{(h)}\left(\bar{x}^{2}-2 \frac{\theta_{1}^{2} \theta_{2}^{2}}{\theta_{1}^{2}+\theta_{2}^{2}}\right) .
$$


Bias and MSE of these estimates are

$$
\operatorname{bias}\left(\tilde{\xi}_{(h)}^{(i)}\right)=\theta^{2}\left(r\left(1-\alpha_{(h)}\right)+\frac{\alpha_{(h)}}{n}\right)
$$

and

$$
\operatorname{MSE}\left(\tilde{\xi}_{(h)}^{(i)}\right)=\theta^{4}\left(r^{2}+\alpha_{(h)}^{2} \zeta_{1}^{*}+2 r \alpha_{(h)} \zeta_{2}^{*}\right)
$$

where

$$
\begin{gathered}
\zeta_{1}^{*}=\frac{1}{n^{3}}(n+1)(n+2)(n+3)-\frac{2}{n}(r+1)(n+1)+(r+1)^{2}, \quad \zeta_{2}^{*}=\frac{1}{n}-r, \\
r=\frac{A G H(a, b)}{\theta^{2}}-1 .
\end{gathered}
$$

Replacing $\bar{x}$ by $\bar{x}_{t}$ in (6), we define the class of estimators

$$
\hat{\xi}_{(h, t)}^{(i)}=A G H(a, b)+\alpha_{(h)}\left\{\bar{x}_{t}^{2}-A G H(a, b)\right\},
$$

for the variance in the presence of large true observations, where $\alpha_{(h)}$ and $\bar{x}_{t}$ are respectively given by (4) and (5). Also here $m$ denotes the number of observations less than a predetermined cutoff point $t$ and follows a binomial distribution with parameters $n$ and $p$, where $p=P(x<t)=1-\exp (-t / \theta)$ and $q=1-p=\exp (-t / \theta)$.

The necessary expectations are

$$
\mathrm{E}\left(\bar{x}_{t}\right)=p \theta, \quad \mathrm{E}\left(\bar{x}_{t}^{2}\right)=\frac{\theta^{2}}{n}\left(\lambda+n p^{2}\right), \quad \mathrm{E}\left(\bar{x}_{t}^{4}\right)=\frac{\theta^{4}}{n^{3}}\left(\eta_{1}+\eta_{2}\right),
$$

where

$$
\begin{aligned}
\eta_{1} & =3 \lambda^{2}(n-2)+6 \lambda\left(n^{2} p^{2}+2 n p q+2\right), \\
\eta_{2} & =n p^{3}\left(n^{2} p+4 q+8\right)-12 n p q(t / \theta)^{2}-4 q(t / \theta)^{3}, \\
\lambda & =1-2 q t / \theta-q^{2} .
\end{aligned}
$$

Bias and MSE of the estimates defined in (8) are

$$
\operatorname{bias}\left(\hat{\xi}_{(h, t)}^{(i)}\right)=\theta^{2}\left(r\left(1-\alpha_{(h)}\right)+\frac{\alpha_{(h)}}{n}\left(\lambda+n p^{2}-n\right)\right)
$$

and

$$
\begin{aligned}
\operatorname{MSE}\left(\hat{\xi}_{(h, t)}^{(i)}\right)= & r^{2} \theta^{4}+\alpha_{(h)}^{2}\left(\mathrm{E}\left(\bar{x}_{t}^{4}\right)+A G H^{2}(a, b)-2 A G H(a, b) \mathrm{E}\left(\bar{x}_{t}^{2}\right)\right) \\
& +2 r \theta^{2} \alpha_{(h)}\left(\mathrm{E}\left(\bar{x}_{t}^{2}\right)-A G H(a, b)\right) .
\end{aligned}
$$

Substituting (9) in (10) gives

$$
\operatorname{MSE}\left(\hat{\xi}_{(h, t)}^{(i)}\right)=\theta^{4}\left(r^{2}+\alpha_{(h)}^{2} \varepsilon_{1}+2 r \alpha_{(h)} \varepsilon_{2}\right)
$$

where

$$
\begin{aligned}
\varepsilon_{1} & =\frac{1}{n^{3}}\left(\eta_{1}+\eta_{2}\right)+(r+1)^{2}-\frac{2}{n}(r+1)\left(\lambda+n p^{2}\right) \\
\varepsilon_{2} & =\frac{1}{n}\left(\lambda+n p^{2}\right)-(r+1) .
\end{aligned}
$$


As $t \rightarrow \infty$, we get $p \rightarrow 1, q \rightarrow 0$, and thus $\operatorname{MSE}\left(\hat{\xi}_{(h, t)}^{(i)}\right) \rightarrow \operatorname{MSE}\left(\tilde{\xi}_{(h)}^{(i)}\right)$. From (7) and (10) we have

$$
\begin{aligned}
\operatorname{MSE}\left(\hat{\xi}_{(h, t)}^{(i)}\right)-\operatorname{MSE}\left(\tilde{\xi}_{(h)}^{(i)}\right) & =\theta^{4}\left(\alpha_{(h)}^{2}\left(\varepsilon_{1}-\zeta_{1}^{*}\right)+2 r \alpha_{(h)}\left(\varepsilon_{2}-\zeta_{2}^{*}\right)\right) \\
& =\frac{\theta^{4} \alpha_{(h)}}{n^{3}}\left(\alpha_{(h)}\left(X+2 n^{2} X^{*}\right)-2 r X^{*}\left(1-\alpha_{(h)}\right) n^{2}\right) \\
& <0,
\end{aligned}
$$

if

or if

$$
r \geq \frac{\alpha_{(h)}\left(2 n^{2} X^{*}+X\right)}{2 X^{*}\left(1-\alpha_{(h)}\right) n^{2}},
$$

or if

$$
\frac{A G H(a, b)}{\theta^{2}} \geq \frac{\alpha_{(h)}\left(2 n^{2} X^{*}+X\right)}{2 n^{2} X^{*}\left(1-\alpha_{(h)}\right)}
$$

where

$$
0 \leq \theta^{2} \leq \frac{A G H(a, b) 2 n^{2} X^{*}\left(1-\alpha_{(h)}\right)}{\alpha_{(h)}\left(2 n^{2} X^{*}+X\right)}
$$

$$
X=\left(\eta_{1}+\eta_{2}\right)-(n+1)(n+2)(n+3), \quad X^{*}=n\left(1-p^{2}\right)-\lambda+1 .
$$

Thus we established the following theorem:

Theorem 2.1: The classes of shrinkage estimators $\hat{\xi}_{(h, t)}^{(i)}$ are more efficient than $\tilde{\xi}_{(h)}^{(i)}, i=$ $1,2,3$, if $\theta^{2}$ is between 0 and $2 n^{2} X^{*} A G H(a, b)\left(1-\alpha_{(h)}\right) /\left(\alpha_{(h)}\left(2 n^{2} X^{*}+X\right)\right)$.

\section{Special Cases}

For $h=1$ in (6) we get classes of shrinkage estimators $\tilde{\psi}_{(1)}^{(i)}, i=1,2,3$, for $\theta^{2}$ as

$$
\tilde{\psi}_{(1)}^{(i)}=A G H(a, b)+\alpha_{(1)}\left(\bar{x}^{2}-A G H(a, b)\right),
$$

where $\alpha_{(1)}=n^{2} /(n+2)(n+3)$. The MSE of $\tilde{\psi}_{(1)}^{(i)}$ can be easily obtained by putting $h=1$ in (10) and is given as

$$
\operatorname{MSE}\left(\tilde{\psi}_{(1)}^{(i)}\right)=\theta^{4}\left(r^{2}+\alpha_{(1)}^{2} \zeta_{1}^{*}+2 r \alpha_{(1)} \zeta_{2}^{*}\right)
$$

For $h=1$ in (8) we get shrinkage estimators in the presence of large true observations as

$$
\hat{\psi}_{(1, t)}^{(i)}=A G H(a, b)+\alpha_{(1)}\left(\bar{x}_{t}^{2}-A G H(a, b)\right) .
$$

Putting $h=1$ in $(11)$, we get

$$
\operatorname{MSE}\left(\hat{\psi}_{(1, t)}^{(i)}\right)=\theta^{4}\left(r^{2}+\alpha_{(1)}^{2} \varepsilon_{1}+2 r \alpha_{(1)} \varepsilon_{2}\right) .
$$

As $t \rightarrow \infty$, we get $p \rightarrow 1, q \rightarrow 0$, and $\operatorname{MSE}\left(\hat{\psi}_{(1, t)}^{(i)}\right) \rightarrow \operatorname{MSE}\left(\tilde{\psi}_{(1)}^{(i)}\right)$. Thus we proved the following theorem:

Theorem 2.2: The classes of shrinkage estimators $\hat{\psi}_{(1, t)}^{(i)}$ are more efficient than $\tilde{\psi}_{(1)}^{(i)}$, $i=1,2,3$, if $\theta^{2}$ is between 0 and $2 X^{*} A G H(a, b)(5 n+6) /\left(2(n+2)(n+3) X^{*}+X\right)$. 


\section{Numerical Illustrations and Comparisons}

To have a concrete idea about the performance of the proposed estimators $\hat{\psi}_{(1, t)}^{(i)}$, we have computed their percentage relative efficiency (PRE) with respect to $\hat{\theta}_{\text {MMSE }}$ given in (2) and $\tilde{\psi}_{(1)}^{(i)}$ given by (12) for various values of $n, \theta_{1}^{2} / \theta^{2}, \theta_{2}^{2} / \theta^{2}$, and for different cutoff points $t$. The following formulae are used for this calculation:

$\operatorname{PRE}\left(\hat{\psi}_{(1, t)}^{(i)}, \hat{\theta}_{\mathrm{MMSE}}\right)=100 \frac{\operatorname{MSE}\left(\hat{\theta}_{\mathrm{MMSE}}\right)}{\operatorname{MSE}\left(\hat{\psi}_{(1, t)}^{(i)}\right)}, \quad \operatorname{PRE}\left(\hat{\psi}_{(1, t)}^{(i)}, \tilde{\psi}_{(1)}^{(i)}\right)=100 \frac{\operatorname{MSE}\left(\tilde{\psi}_{(1)}^{(i)}\right)}{\operatorname{MSE}\left(\hat{\psi}_{(1, t)}^{(i)}\right)}$,

where $\operatorname{MSE}\left(\hat{\theta}_{\text {MMSE }}\right), \operatorname{MSE}\left(\tilde{\psi}_{(1)}^{(i)}\right)$, and $\operatorname{MSE}\left(\hat{\psi}_{(1, t)}^{(i)}\right)$ are given by (3), (13) and (15).

From Table 1 we observe that the suggested classes of estimators show better efficiency than $\hat{\theta}_{\text {MMSE }}$ if

- $t / \theta=1, n \leq 11$, and $\Delta \in[0.7,2.7]$, where $\Delta=A G H(0,1) / \theta^{2}$,

- $2 \leq t / \theta \leq 10, n \leq 30$, and $\Delta \in[0.3,2.0]$.

From extended computation and from Table 1, we further observe that

- for $\Delta<1, \hat{\psi}_{(1, t)}^{(1)}$ (based on the AM) is more efficient than $\hat{\theta}_{\mathrm{MMSE}}, \hat{\psi}_{(1, t)}^{(2)}$, and $\hat{\psi}_{(1, t)}^{(3)}$,

- for $\Delta>1, \hat{\psi}_{(1, t)}^{(3)}$ (based on the HM) is more efficient than $\hat{\theta}_{\mathrm{MMSE}}, \hat{\psi}_{(1, t)}^{(2)}$, and $\hat{\psi}_{(1, t)}^{(1)}$,

- for $\Delta=1$ and $1 \leq t / \theta \leq 3, \hat{\psi}_{(1, t)}^{(1)}$ shows higher efficiency than $\hat{\theta}_{\mathrm{MMSE}}, \hat{\psi}_{(1, t)}^{(2)}$, and $\hat{\psi}_{(1, t)}^{(3)}$, whereas for $\Delta=1$ and $3<t / \theta \leq 10, \hat{\psi}_{(1, t)}^{(3)}$ is better than $\hat{\theta}_{\mathrm{MMSE}}, \hat{\psi}_{(1, t)}^{(2)}$, and $\hat{\psi}_{(1, t)}^{(1)}$.

Further, it reveals from Table 2 that $\hat{\psi}_{(1, t)}^{(i)}$ shows better efficiency than $\tilde{\psi}_{(1)}^{(i)}$ if

- $t / \theta=1, n \leq 5$, and $\Delta \in[0.7,2.0]$, where $\Delta=A G H(0,1) / \theta^{2}$,

- $t / \theta=1,5<n \leq 25$, and $\Delta \in[1.2,2.5]$,

- $2 \leq t / \theta<6, n \leq 25$, and $\Delta \in[0.7,2.0]$.

It is also observed that

- for $\Delta \leq 1$, the estimator $\hat{\psi}_{(1, t)}^{(1)}$ (based on the AM) is more efficient than $\tilde{\psi}_{(1)}^{(i)}, \hat{\psi}_{(1, t)}^{(2)}$, and $\hat{\psi}_{(1, t)}^{(3)}$,

- for $\Delta>1$, the estimator $\hat{\psi}_{(1, t)}^{(3)}$ (based on the HM) is more efficient than $\tilde{\psi}_{(1)}^{(i)}, \hat{\psi}_{(1, t)}^{(2)}$, and $\hat{\psi}_{(1, t)}^{(1)}$,

- for $t / \theta \geq 6$, the classes of estimators $\hat{\psi}_{(1, t)}^{(i)}$ and $\tilde{\psi}_{(1)}^{(i)}$ are equally efficient. 
Table 1: Percentage relative efficiencies, PRE, of the estimators $\hat{\psi}_{(1, t)}^{(i)}, i=1,2,3$, with respect to $\hat{\theta}_{\text {MMSE }}$ for different $n, \theta_{1}^{2} / \theta^{2}, \theta_{2}^{2} / \theta^{2}, t / \theta$.

\begin{tabular}{|c|c|c|c|c|c|c|c|c|c|}
\hline & \multicolumn{3}{|c|}{$t / \theta=1$} & \multicolumn{3}{|c|}{$t / \theta=5$} & \multicolumn{3}{|c|}{$t / \theta=10$} \\
\hline$\left(\frac{\theta_{1}^{2}}{\theta^{2}}, \frac{\theta_{2}^{2}}{\theta^{2}}\right)$ & $n=5$ & $n=15$ & $n=25$ & $n=5$ & $n=15$ & $n=25$ & $n=5$ & $n=15$ & $n=25$ \\
\hline \multicolumn{10}{|l|}{$(0.2,1.0)$} \\
\hline$i=1$ & 196.25 & 71.86 & 43.70 & 199.45 & 137.90 & 125.67 & 175.00 & 125.33 & 115.22 \\
\hline$i=2$ & 143.04 & 62.42 & 39.92 & 175.65 & 131.75 & 122.03 & 157.48 & 120.94 & 112.78 \\
\hline$i=3$ & 116.22 & 56.53 & 37.40 & 156.25 & 125.93 & 118.53 & 142.36 & 116.51 & 110.24 \\
\hline \multicolumn{10}{|l|}{$(0.4,1.2)$} \\
\hline$i=1$ & 324.66 & 87.80 & 49.52 & 217.85 & 142.09 & 128.24 & 187.01 & 127.72 & 116.48 \\
\hline$i=2$ & 244.23 & 78.67 & 46.27 & 210.45 & 140.45 & 127.19 & 182.54 & 126.95 & 116.09 \\
\hline$i=3$ & 196.25 & 71.86 & 43.70 & 199.45 & 137.90 & 125.67 & 175.00 & 125.33 & 115.22 \\
\hline \multicolumn{10}{|l|}{$(0.5,1.3)$} \\
\hline$i=1$ & 440.46 & 97.81 & 52.88 & 218.50 & 142.28 & 128.49 & 186.50 & 127.36 & 116.25 \\
\hline$i=2$ & 330.49 & 88.38 & 49.72 & 218.07 & 142.14 & 128.27 & 187.11 & 127.73 & 116.48 \\
\hline$i=3$ & 263.08 & 81.03 & 47.13 & 213.11 & 141.04 & 127.56 & 184.25 & 127.28 & 116.26 \\
\hline \multicolumn{10}{|l|}{$(0.6,1.4)$} \\
\hline$i=1$ & 627.90 & 109.61 & 56.58 & 213.00 & 141.17 & 128.04 & 181.53 & 125.95 & 115.44 \\
\hline$i=2$ & 465.26 & 99.62 & 53.46 & 218.00 & 142.19 & 128.47 & 185.97 & 127.20 & 116.15 \\
\hline$i=3$ & 364.91 & 91.61 & 50.83 & 218.86 & 142.33 & 128.42 & 187.36 & 127.70 & 116.46 \\
\hline \multicolumn{10}{|l|}{$(0.7,1.5)$} \\
\hline$i=1$ & 955.23 & 123.64 & 60.68 & 202.22 & 138.81 & 126.90 & 172.79 & 123.58 & 114.09 \\
\hline$i=2$ & 691.77 & 112.84 & 57.55 & 210.77 & 140.70 & 127.82 & 179.69 & 125.45 & 115.16 \\
\hline$i=3$ & 530.70 & 104.00 & 54.85 & 216.23 & 141.84 & 128.33 & 184.31 & 126.72 & 115.88 \\
\hline \multicolumn{10}{|l|}{$(0.8,1.6)$} \\
\hline$i=1$ & 1579.4 & 140.51 & 65.25 & 187.71 & 135.33 & 125.11 & 161.34 & 120.34 & 112.22 \\
\hline$i=2$ & 1107.6 & 128.59 & 62.06 & 197.99 & 137.83 & 126.41 & 169.44 & 122.65 & 113.55 \\
\hline$i=3$ & 823.6 & 118.68 & 59.27 & 206.31 & 139.73 & 127.36 & 176.06 & 124.47 & 114.60 \\
\hline \multicolumn{10}{|l|}{$(0.9,1.7)$} \\
\hline$i=1$ & 2845.7 & 160.99 & 70.34 & 171.18 & 130.89 & 122.71 & 148.36 & 116.39 & 109.89 \\
\hline$i=2$ & 1946.6 & 147.60 & 67.06 & 181.75 & 133.79 & 124.29 & 156.67 & 118.96 & 111.41 \\
\hline$i=3$ & 1394.9 & 136.32 & 64.15 & 191.30 & 136.22 & 125.58 & 164.16 & 121.16 & 112.69 \\
\hline \multicolumn{10}{|l|}{$(1.0,2.2)$} \\
\hline$i=1$ & 3352.8 & 257.45 & 89.71 & 122.30 & 113.88 & 112.69 & 109.16 & 101.74 & 100.78 \\
\hline$i=2$ & 5626.4 & 211.84 & 81.33 & 140.32 & 120.96 & 117.02 & 123.80 & 107.78 & 104.64 \\
\hline$i=3$ & 4410.2 & 179.38 & 74.55 & 158.38 & 127.06 & 120.57 & 138.23 & 113.04 & 107.88 \\
\hline \multicolumn{10}{|l|}{$(1.0,2.8)$} \\
\hline$i=1$ & 697.80 & 466.93 & 118.19 & 85.47 & 95.18 & 100.16 & 78.32 & 85.83 & 89.84 \\
\hline$i=2$ & 2146.2 & 293.75 & 95.65 & 140.32 & 120.96 & 117.02 & 100.68 & 97.84 & 98.21 \\
\hline$i=3$ & 5687.1 & 208.65 & 80.69 & 141.87 & 121.52 & 117.35 & 125.05 & 108.26 & 104.94 \\
\hline
\end{tabular}


Table 2: Percentage relative efficiencies, PRE, of the estimators $\hat{\psi}_{(1, t)}^{(i)}$ with respect to $\tilde{\psi}_{(1)}^{(i)}$, $i=1,2,3$, for different $n, \theta_{1}^{2} / \theta^{2}, \theta_{2}^{2} / \theta^{2}, t / \theta$.

\begin{tabular}{|c|c|c|c|c|c|c|c|c|c|}
\hline & \multicolumn{3}{|c|}{$t / \theta=1$} & \multicolumn{3}{|c|}{$t / \theta=5$} & \multicolumn{3}{|c|}{$t / \theta=10$} \\
\hline$\left(\frac{\theta_{1}^{2}}{\theta^{2}}, \frac{\theta_{2}^{2}}{\theta^{2}}\right)$ & $n=5$ & $n=15$ & $n=25$ & $n=5$ & $n=15$ & $n=25$ & $n=5$ & $n=15$ & $n=25$ \\
\hline \multicolumn{10}{|l|}{$(0.2,1.0)$} \\
\hline$i=1$ & 112.51 & 57.44 & 37.98 & 114.35 & 110.22 & 109.22 & 100.33 & 100.17 & 100.14 \\
\hline$i=2$ & 91.10 & 51.70 & 35.44 & 111.87 & 109.12 & 108.34 & 100.29 & 100.16 & 100.14 \\
\hline$i=3$ & 81.85 & 48.60 & 33.97 & 110.05 & 108.25 & 107.66 & 100.26 & 100.15 & 100.13 \\
\hline \multicolumn{10}{|l|}{$(0.4,1.2)$} \\
\hline$i=1$ & 174.25 & 68.87 & 42.58 & 116.92 & 111.45 & 110.26 & 100.37 & 100.19 & 100.15 \\
\hline$i=2$ & 134.27 & 62.08 & 39.92 & 115.70 & 110.83 & 109.73 & 100.35 & 100.18 & 100.15 \\
\hline$i=3$ & 112.51 & 57.44 & 37.98 & 114.35 & 110.22 & 109.22 & 100.33 & 100.17 & 100.14 \\
\hline \multicolumn{10}{|l|}{$(0.5,1.3)$} \\
\hline$i=1$ & 237.05 & 76.94 & 45.56 & 117.60 & 111.93 & 110.70 & 100.37 & 100.19 & 100.15 \\
\hline$i=2$ & 177.28 & 69.32 & 42.75 & 116.98 & 111.49 & 110.29 & 100.37 & 100.19 & 100.15 \\
\hline$i=3$ & 143.29 & 63.78 & 40.60 & 116.08 & 111.01 & 109.88 & 100.36 & 100.18 & 100.15 \\
\hline \multicolumn{10}{|l|}{$(0.6,1.4)$} \\
\hline$i=1$ & 347.16 & 87.19 & 49.09 & 117.76 & 112.29 & 111.09 & 100.37 & 100.19 & 100.15 \\
\hline$i=2$ & 251.11 & 78.47 & 46.10 & 117.66 & 112.00 & 110.77 & 100.37 & 100.19 & 100.15 \\
\hline$i=3$ & 195.49 & 71.87 & 43.71 & 117.25 & 111.66 & 110.44 & 100.37 & 100.19 & 100.15 \\
\hline \multicolumn{10}{|l|}{$(0.7,1.5)$} \\
\hline$i=1$ & 554.77 & 100.24 & 53.27 & 117.44 & 112.54 & 111.41 & 100.35 & 100.19 & 100.16 \\
\hline$i=2$ & 386.38 & 90.12 & 50.06 & 117.73 & 112.36 & 111.17 & 100.36 & 100.19 & 100.16 \\
\hline$i=3$ & 289.00 & 82.23 & 47.41 & 117.75 & 112.14 & 110.92 & 100.37 & 100.19 & 100.15 \\
\hline \multicolumn{10}{|l|}{$(0.8,1.6)$} \\
\hline$i=1$ & 982.20 & 116.97 & 58.23 & 116.73 & 112.66 & 111.66 & 100.33 & 100.19 & 100.16 \\
\hline$i=2$ & 655.96 & 105.05 & 54.74 & 117.26 & 112.59 & 111.49 & 100.35 & 100.19 & 100.16 \\
\hline$i=3$ & 469.49 & 95.53 & 51.80 & 117.60 & 112.47 & 111.31 & 100.36 & 100.19 & 100.16 \\
\hline \multicolumn{10}{|l|}{$(0.9,1.7)$} \\
\hline$i=1$ & 1924.3 & 138.58 & 64.10 & 115.75 & 112.67 & 111.84 & 100.31 & 100.18 & 100.16 \\
\hline$i=2$ & 1246.6 & 124.31 & 60.29 & 116.39 & 112.68 & 111.73 & 100.33 & 100.19 & 100.16 \\
\hline$i=3$ & 852.58 & 112.73 & 57.01 & 116.92 & 112.64 & 111.61 & 100.34 & 100.19 & 100.16 \\
\hline \multicolumn{10}{|l|}{$(1.0,2.2)$} \\
\hline$i=1$ & 3078.7 & 253.49 & 89.15 & 112.30 & 112.13 & 111.99 & 100.24 & 100.17 & 100.15 \\
\hline$i=2$ & 4556.9 & 196.90 & 77.84 & 113.64 & 112.42 & 112.00 & 100.27 & 100.18 & 100.15 \\
\hline$i=3$ & 3199.9 & 158.98 & 69.21 & 114.91 & 112.61 & 111.93 & 100.29 & 100.18 & 100.16 \\
\hline \multicolumn{10}{|l|}{$(1.0,2.8)$} \\
\hline$i=1$ & 892.52 & 544.85 & 131.73 & 109.33 & 111.06 & 111.64 & 100.18 & 100.15 & 100.14 \\
\hline$i=2$ & 2136.5 & 300.72 & 97.54 & 111.50 & 111.90 & 111.94 & 100.22 & 100.17 & 100.15 \\
\hline$i=3$ & 4560.1 & 193.07 & 77.01 & 113.76 & 112.44 & 111.99 & 100.27 & 100.18 & 100.15 \\
\hline
\end{tabular}




\section{Conclusion}

From the above we conclude that the developed classes of estimators $\hat{\psi}_{(1, t)}^{(i)}, i=1,2,3$, are to be preferred over $\hat{\theta}_{\text {MMSE }}$ and $\tilde{\psi}_{(1)}^{(i)}$ in practice as they are more efficient than $\hat{\theta}_{\text {MMSE }}$ and $\tilde{\psi}_{(1)}^{(i)}$ with larger gain in efficiency.

We also note that $\hat{\psi}_{(1, t)}^{(1)}$ (based on the AM) performed better than $\hat{\theta}_{\mathrm{MMSE}}, \hat{\psi}_{(1, t)}^{(2)}, \hat{\psi}_{(1, t)}^{(3)}$, and $\tilde{\psi}_{(1)}^{(i)}, i=1,2,3$, when $t / \theta \leq 10, n \leq 25$, and $0.7 \leq \Delta<1.0$, while for $t / \theta \geq 6$, $n \leq 25$, and $0.7 \leq \Delta<1.0$, the proposed estimators $\hat{\psi}_{(1, t)}^{(1)}$ and $\tilde{\psi}_{(1)}^{(1)}$ are equally efficient, so one can choose any one of them. It is further observed that the suggested estimator $\hat{\psi}_{(1, t)}^{(3)}$ (based on the HM) has smaller MSE than $\hat{\theta}_{\mathrm{MMSE}}, \hat{\psi}_{(1, t)}^{(1)}, \hat{\psi}_{(1, t)}^{(2)}$, and $\tilde{\psi}_{(1)}^{(i)}, i=1,2,3$, when $t / \theta<6, n \leq 25$, and $1 \leq \Delta<2$. In such situations we suggest the use of the estimator $\hat{\psi}_{(1, t)}^{(3)}$. On the other hand it is noted that for $t / \theta \geq 6, n \leq 25$, and $0.7 \leq \Delta<1$ the estimators $\hat{\psi}_{(1, t)}^{(3)}$ and $\tilde{\psi}_{(1)}^{(3)}$ approximately have the same MSE and hence any one of them can be chosen in such situation.

\section{Acknowledgements}

The authors are grateful to the editor Professor Herwig Friedl and the learned referee for their valuable suggestions regarding the improvement of the paper.

\section{References}

Bartholomew, D. J. (1957). A problem of life testing. Journal of the American Statistical Association, 65, 350-355.

Jani, P. N. (1991). A class of shrinkage estimators for the scale parameter of the exponential distribution. IEEE Transactions on Reliability, 40, 68-70.

Mehta, J. S., and Srinivasan, R. (1971). Estimation of the mean by shrinkage to a point. Journal of the American Statistical Association, 66, 86-90.

Ojha, V. P. (1982). A note on estimation of variance in exponential density. Journal of the Indian Society of Agricultural Statistics, 34, 82-88.

Ojha, V. P., and Srivastava, S. R. (1979). An estimator of the population variance in the presence of large true observations. Journal of the Indian Society of Agricultural Statistics, 31, 77-84.

Pandey, B. N., and Singh, J. (1977). A note on estimation of variance in exponential density. Sankhya, B, 39, 294-298.

Saxena, S. (2006). A decision theoretic estimation in exponential product life testing model using guesstimate. Vikalpa, 31, 31-45.

Saxena, S., and Singh, H. P. (2004). Estimating various measures in normal population through a single class of estimators. Journal of the Korean Statistical Society, 33, 323-337.

Saxena, S., and Singh, H. P. (2006). From ordinary to shrinkage square-root estimators. Communications in Statistics - Theory and Methods, 35, 1037-1058. 
Searls, D. T. (1966). An estimators for a population mean which reduces the effect of large true observations. Journal of the American Statistical Association, 59, 1200-1204.

Singh, H. P. (1987). A modified estimator for normal population variance in the presence of large true observations. Gujarat Statistical Review, 14, 15-30.

Singh, H. P., and Chander, V. (2007). Some classes of shrinkage estimators for estimating the scale parameter towards an interval of exponential distribution. Journal of Probability and Statistical Science. (accepted)

Singh, H. P., and Saxena, S. (2003). An improved class of shrinkage estimators for the variance of a normal population. Statistics in Transition, 6, 119-129.

Singh, H. P., and Saxena, S. (2005). Using prior information in estimation of $k^{\text {th }}$ exponent of scale parameter in negative exponent population. Statistical Methods, 7, 116126.

Singh, H. P., Saxena, S., and Espejo, M. R. (2004). Estimation of standard deviation in normal parent by shrinkage towards an interval. Journal of Statistical Planning and Inference, 126, 479-493.

Singh, H. P., and Shukla, S. K. (2002). A class of shrinkage estimators for the variance of exponential distribution with type-I censoring. Indian Association for Productivity, Quality and Reliability Transactions, 27, 119-141.

Srivastava, R. S. (1986). On estimation of mean life time from a time censored sample using exponential failure model. Journal of National Academy of Mathematics, India, 4, 107-113.

Srivastava, R. S., and Kumar, G. (1990). Efficient estimation of variance of exponential population in the presence of large true observations. Journal of National Academy of Mathematics, India, 8, 61-71.

Srivastava, R. S., Pandey, B. N., and Srivastava, S. R. (1985). A modified estimator for the population mean which reduces the effect of large true observations. Journal of the Indian Society of Agricultural Statistics, 37, 71-78.

Thompson, J. R. (1968). Accuracy borrowing in the estimation of mean by shrinkage to an interval. Journal of the American Statistical Association, 63, 113-122.

Tracy, D. S., Singh, H. P., and Raghuvanshi, H. S. (1996). Some shrinkage estimator for the variance of exponential density. Microelectron Reliability, 36, 651-655.

Upadhyaya, L. N., Gangele, R. K., and Singh, H. P. (1997). A shrinkage estimator for the scale parameter of the exponential distribution with type-I censoring. International Journal of Management System, 13, 103-114.

Authors Address:

Housila P. Singh and Vankim Chander

School of Studies in Statistics

Vikram University

Ujjain - 456010, M. P.

India

E-mail: hpsujn@rediffmail.com and vcupadhyay06@yahoo.co.in 